\title{
Serum Cholesterol Levels and the Ratio of Polyunsaturated to Saturated Fatty Acid as an Indicator of Lipid Metabolism in Rat Liver
}

\author{
Kazuko HiraI, ${ }^{1}$ Takayo NaKano, ${ }^{1}$ Yoshiho KatAyama, ${ }^{1}$ \\ and Shizuko AMAGASE ${ }^{2}$ \\ ${ }^{1}$ Department of Food and Nutrition, Faculty of the Science of Living, \\ Osaka City University, Sumiyoshi-ku, Osaka 558, Japan \\ ${ }^{2}$ Institute for Molecular and Cellular Biology, Osaka University, \\ Suita, Osaka 565, Japan \\ (Received June 9, 1984)
}

\begin{abstract}
Summary Tne dietary effects of phytosterol and polyunsaturated fat (PUFA-fat) on the cholesterol and fatty acids levels in serum and liver of female rats were studied, with the following results. (1) Addition of $1 \%$ cholesterol with $0.25 \%$ cholic acid to a $10 \%$ butter diet increased cholesterol levels and decreased the ratio of polyunsaturated fatty acid (PUFA) to saturated fatty acid (SFA) in the serum and liver. These changes diminished upon additions of phytosterol and PUFA-fat (cod liver oil) to the $10 \%$ butter-cholesterol diet. (2) While the cholesterol-rich diet increased the fatty acid content of the liver, addition of phytosterol to the butter-cholesterol diet depressed this increase. On the other hand, addition of PUFA-fat to the butter-cholesterol diet caused an increase of total fatty acid in the liver. (3) A negative relationship was found between the cholesterol level and the ratio of PUFA to SFA in serum $(r=-0.810)$ and also in liver $(r=-0.548)$. There were significant positive correlations between the values for the serum and liver cholesterol levels $(r=0.720)$ and also between the respective values in the ratios of PUFA to SFA $(r=0.521)$. These results indicate that dietary phytosterol and PUFA-fat modulate the levels of cholesterol in proportion to the increase in the ratio of PUFA to SFA in both serum and liver with the cholesterol diet, and show that the changes in the values for the serum reflected in changes in the liver.
\end{abstract}

Key Words PUFA/SFA ratio, correlation between serum and liver cholesterol and PUFA/SFA ratio

An increased level of serum cholesterol, which is known as a coronary risk factor in humans $(1,2)$, is influenced by a variety of dietary factors $(3,4)$. Tallow raises the serum cholesterol level while vegetable oils lower it $(5,6)$. The hy-

1 平井和子, 中野貴世, 片山吉穂, ${ }^{2}$ 天ケ瀨 倭文子 
pocholesterolemic effect of certain vegetable oils is due to their high content of polyunsaturated fatty acid (PUFA) and low content of saturated fatty acid (SFA), as serum cholesterol is reduced by an increase in the ratio of PUFA to SFA in $\operatorname{diet}(7,8)$. Toussant et al. have presented evidence for the suppression of fatty acid synthesis in the liver by PUFA (9), and Ramesha et al. have demonstrated that the effect of PUFA on hypercholesterolemia is due to stimulation of biliary and fecal excretion of cholesterol (10). Another hypocholesterolemic factor in vegetable oil is phytosterol $(3,11)$, which is thought to interfere with cholesterol absorption $(12,13)$ and affect cholesterol metabolism in the body $(14,15)$. Fish oil also decreases serum lipid levels in humans (16) and rats (17), which is related to its high PUFA content. We recently found a direct relationship between the concentration of oleic acid in serum and the serum cholesterol level (18). However, the interrelation between the changes in cholesterol levels and fatty acid composition in the serum and liver due to dietary factors is not clear. This communication reports the effects of dietary cholesterol, phytosterol and PUFA-fat (cod liver oil) on the relationships among these variables in serum and liver of female rats.

\section{EXPERIMENTAL PROCEDURE}

Animals. Female Wistar rats (Clea Japan) were housed individually in stainless steel wire-bottom cages and kept under standardized conditions of light (08:00-20:00) and temperature $\left(22^{\circ} \mathrm{C}\right)$. Eleven groups, each comprising four female rats were given the experimental diets ad libitum for 18 days and then made to fast for $17-20 \mathrm{~h}$ prior to autopsy. About $1 \mathrm{~min}$ after anesthetizing the rats with ethyl ether, blood was collected through the abdominal aorta with a syringe and then centrifuged to separate the serum. Livers were removed and stored at $-20^{\circ} \mathrm{C}$ until analyzed. Small pieces of liver tissues were fixed in a $10 \%$ formalin solution for histochemical determination.

Diets. The basal diet (group A) consisted of $10 \%$ butter (Snow Brand Milk Products Co.), $20 \%$ casein, 4\% Harper salt mixture (19), 1\% Harper Vitamin mixture (19), 2,000 IU retinyl palmitate, 1,000 IU cholecalciferol, $0.1 \% \alpha$-tocopherol, $0.15 \%$ choline chloride, and sucrose to $100 \%$. For the cholesterol diet (groups B to K), $1 \%$ cholesterol and $0.25 \%$ cholic acid were added to the basal diet. All the test diets were prepared by addition of phytosterol $(0.02,0.2,2.0,5.0 \%$, groups C, D, E and J) or cod liver oil $(1,3,5,10 \%$, groups F, G, H and K) to the cholesterol diet with proportionate reduction of the amount of sucrose. Phytosterol and cod liver oil were gifts from Riken Vitamin Co., the purity of the sterols being $95 \%$, with the composition of the sterol fraction being $45 \% \beta$-sitosterol, $29 \%$ stigmasterol, $24 \%$ campesterol and $2 \%$ brasicasterol.

Preparation and analysis of cholesterol and fatty acids. Saponification of serum and preparation into sterol and fatty acid fractions were carried out by the Standard Method of the Japan Oil Chemist's Society for Analysis of Fats and Oils (20). The sterol fraction was directly loaded on a gas-liquid chromatograph 
(GLC) for analysis. Fatty acid methyl esters were prepared using $\mathrm{BF}_{3}$-methanol(21) and analyzed in GLC.

Liver lipids were extracted by homogenizing the liver with chloroform methanol $(2 / 1, \mathrm{v} / \mathrm{v})$ and purified as described by Folch et al. (22). The extracted liver lipids fraction was prepared and analyzed for cholesterol and fatty acids as described above.

Gas-liquid chromatography. A gas-liquid chromatograph (Model 163, Hitachi Ltd.) equipped with flame ionization detectors was used for analysis of cholesterol and fatty acid methyl esters (18). Cholesterol was separated on a 3-m glass column ( $3 \mathrm{~mm}$ i.d.) containing $2 \% \mathrm{OV}-17$ on $60-80$ mesh Uniport H.P. (Gasukuro Kogyo) at a column temperature of $250^{\circ} \mathrm{C}$ with nitrogen as the carrier gas $(50 \mathrm{ml} / \mathrm{min})$. For analysis of fatty acid methyl esters, a $3-\mathrm{m}$ glass column ( $3 \mathrm{~mm}$ i.d.) packed with $5 \%$ SP-2300 coated on 60-80 mesh Uniport H.P. was utilized (23). The temperature was programmed from 180 to $240^{\circ} \mathrm{C}$ at $4^{\circ} \mathrm{C}$ increments per min. The carrier gas (nitrogen) flow rate was $17.5 \mathrm{ml} / \mathrm{min}$. Fatty acid methyl esters were identified by comparison of retention data with those of authentic standards and by the equivalent chain length (24). The contents of cholesterol and fatty acids were corrected on the basis of the recovery of the added internal standard of $5 \alpha$-cholestane and heptadecanoic acid $(17: 0)$, respectively.

Histochemical determinations of liver tissues. The small pieces of hepatic tissues already fixed with a formalin solution were formed into blocks by the usual method. Sections ( $5 \mu \mathrm{m}$ thick) were cut from these tissue blocks and stained by Mayer's hematoxylin-eosin staining method or by Daddi's staining method (25). The photomax microscope (LB type, Olympus) was used for histochemical observation and photographing.

Statistical analysis. Data were analyzed by Student's $t$ test.

\section{RESULTS AND DISCUSSION}

\section{Relationship between cholesterol level and fatty acid composition in the serum}

Addition of $1 \%$ cholesterol with $0.25 \%$ cholic acid to the $10 \%$ butter basal diet increased the serum cholesterol concentration from 79 to $733 \mathrm{mg} / 100 \mathrm{ml}$ (group A versus group B, Table 1). The increment in the mean serum cholesterol level was reduced in proportion to the amounts of added phytosterol (groups C, D, E and J) and PUFA-fat (groups F, G, H and K) to the cholesteol diet (group B).These results of the effects of dietary cholesterol, phytosterol and PUFA-fat intakes on the serum cholesterol level were similar to those of earlier observations on rats (11) and humans $(26,27)$.

When $1 \%$ cholesterol with $0.25 \%$ cholic acid was added to the basal diet, the PUFA/SFA ratio in the serum decreased (A vs. B, Fig. 1). The reduction in the PUFA/SFA ratio was less marked by addition of phytosterol and PUFA-fat to the cholesterol diet (B vs. C to H, Fig. 1). The results obtained from individual animals showed an inverse relationship existing between the serum cholesterol level and the 


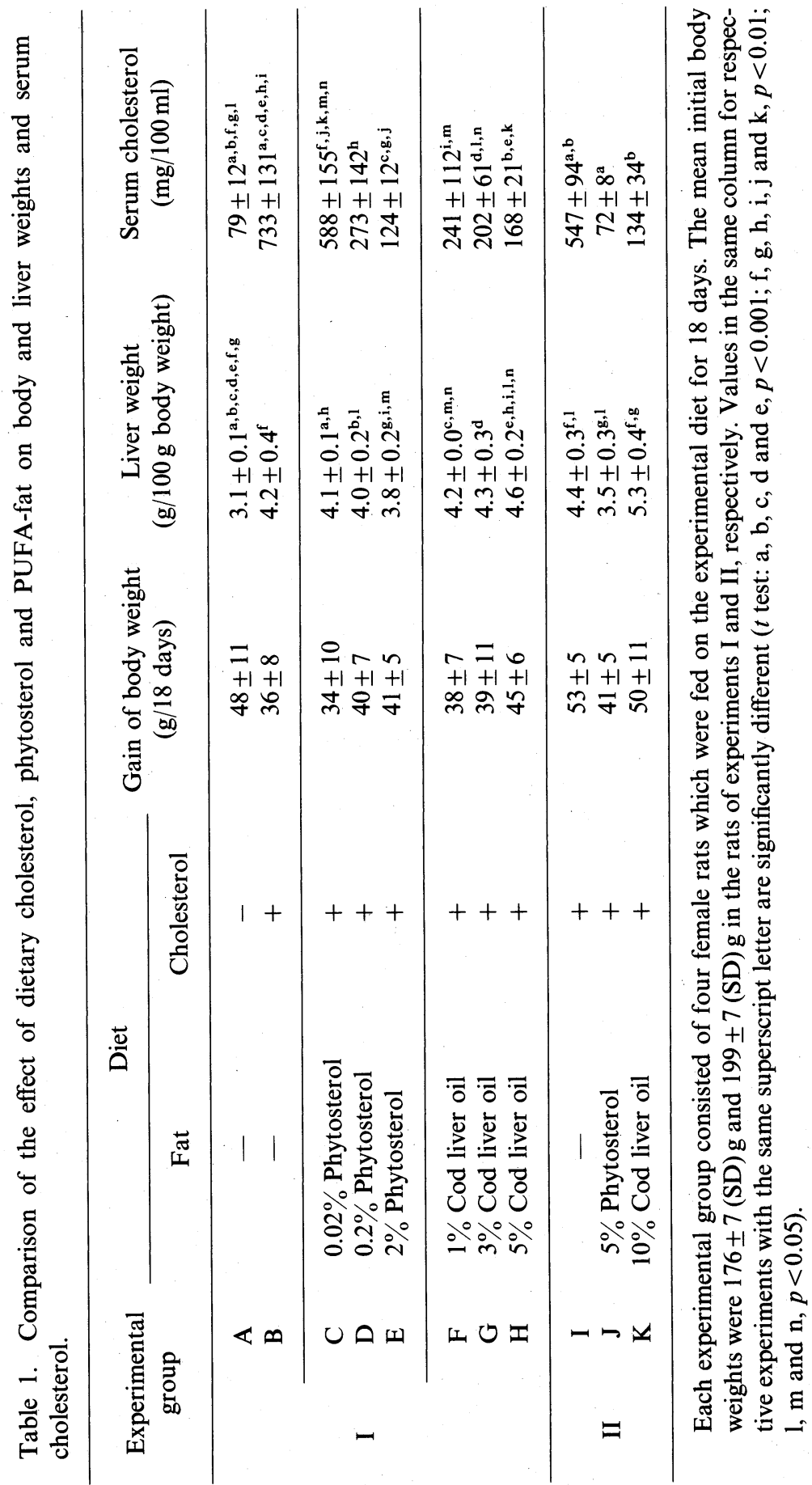

J. Nutr. Sci. Vitaminol. 


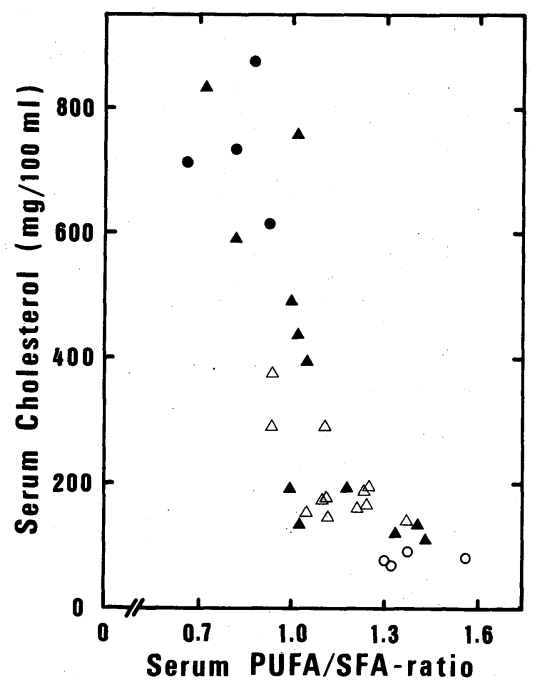

Fig. 1. Relationship of cholesterol level and PUFA/SFA ratio for sera obtained from individual rats fed on different diets. Symbols: rats on cholesterol-free diet, $\bigcirc$, group A in Table 1; rats on cholesterol diet, $\mathbf{O}$, group B; rats on cholesterol-phytosterol diets, $\Delta$, groups C, D and E; rats on cholesterol-cod liver oil diets, $\triangle$, groups F, G and $\mathrm{H}$; for details of diets, see Table $1(r=-0.810, p<0.001)$.

PUFA/SFA ratio in all rats $(r=-0.810$, Fig. 1$)$. This indicates that the levels of serum cholesterol were lower at higher PUFA/SFA ratios of serum lipids and vice versa. Thus, dietary cholesterol intake and the PUFA/SFA ratio were not directly reflected in those values for the serum. These observations suggest the following two manifestations of the hypocholesterolemic effects. One is concerned with an increase of the PUFA/SFA ratio in the serum when dietary phytosterol or PUFA is increased, and the other is related to a decrease in the serum cholesterol level when the PUFA/SFA ratio is elevated. The inverse correlation between the serum cholesterol and the PUFA/SFA ratio in the serum indicates that the increase of the percentage of PUFA in the serum might be due to the increase in compartments of lipoproteins other than the fatty acid moiety of the cholesterol ester.

\section{Relationship between cholesterol level and fatty acid composition in the liver}

As shown in Table 1, the cholesterol diet increased the liver weight and induced fatty livers (group A vs. group B), which was consistent with previous findings (28). Addition of phytosterol at increasing doses to the cholesterol diet reduced the liver weight (B vs. C to E), while addition of PUFA-fat in increasing amounts further increased the liver weight ( $\mathrm{B} v s$. $\mathrm{F}$ to $\mathrm{H}$ ). When expressed in gram $\%$ of body weight, total fatty acid in the liver was higher in the PUFA-fat groups than in the phytosterol groups (Fig. 2). The liver weight was significantly correlated with the total fatty acid in the liver $(r=0.843)$. However, there was no correlation between 


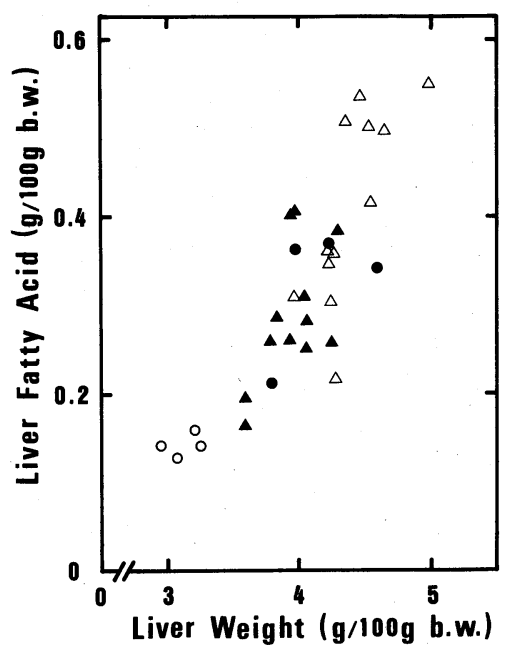

Fig. 2. Relationship between liver fatty acids and weight of liver from rats fed on different diets. For symbols, refer to Fig. $1(r=0.843, p<0.001)$.

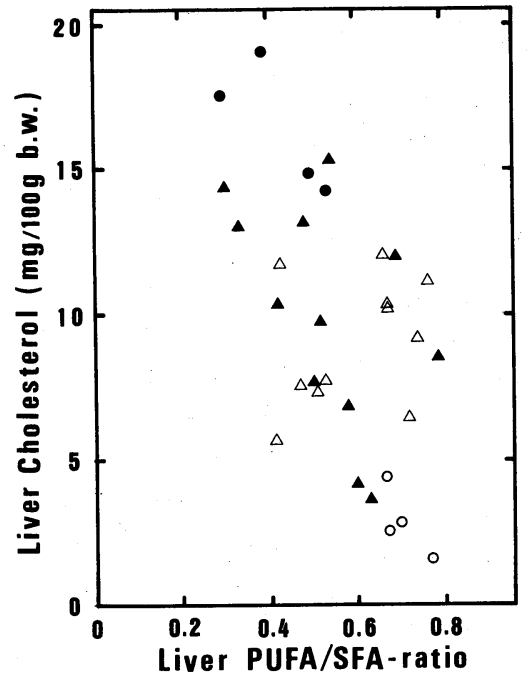

Fig. 3. Relationship between cholesterol level and PUFA/SFA ratio for livers of rats fed on different diets. For symbols, refer to Fig. $1(r=-0.548, p<0.01)$.

total fatty acid and the PUFA/SFA ratio in the liver $(r=-0.057)$. These results show that the PUFA-fat diets might have led to a greater accumulation of fat in the liver than did the PUFA-fat-free diets and that the phytosterol diets reduced the fat in the liver, suggesting that the fatty acid accumulation in the liver was not related to the changes of the PUFA/SFA ratio in the liver. 
The cholesterol diet increased the cholesterol level in the liver as well as in the serum (Table 1 and Figs. 1 and 3). However, an increase in the amounts of dietary phytosterol and PUFA-fat added to the cholesterol diet decreased the liver cholesterol concentration (B vs. C to H, Fig. 3).

As shown in Fig. 3, the PUFA/SFA ratio in the liver was low in the cholesterolfed rats (A vs. B to H). Addition of phytosterol (C, D and E) and PUFA-fat (F, G and $\mathrm{H}$ ) to the cholesterol diet induced changes in the fatty acid composition of the liver and resulted in an increase in the PUFA/SFA ratio. The cholesterol level versus PUFA/SFA ratio in the liver. revealed an significant inverse relationship $(r=-0.548)$, this indicating that the increase in the PUFA/SFA ratio corresponded to the decrease in the liver cholesterol level. However, there was no relationship between the PUFA/SFA ratio and total fatty acids $(r=-0.057)$ as described above. Therefore, these results suggest that changes in the composition of fatty acids rather than in the total amount of fatty acids in the liver, play a greater role in cholesterol metabolism, and that the changes in the PUFA/SFA ratio do not have any effect on the deposits of total fatty acid in liver.

\section{Light microscopic observation of liver tissues from rats on the experimental diets}

As shown in Photo 1, lipid droplets were found in the periportal regions of the hepatic acini of rats on the cholesterol diet (group I in Table 1). These droplets varied in size and the hepacyte nuclei were relatively intact although some showed pyknotic changes. These findings were consistent with those for the fatty liver (Fig. 2). On the other hand, rats on the phytosterol-supplemented cholesterol diet (group $\mathrm{j}$ in Table 1) showed a relatively intact liver without lipid deposition and no nuclear change as shown in Photos 2 and 3. In contrast, rats on the PUFA-fatsupplemented cholesterol diet (group K in Table 1) showed marked lipid deposits in many hepatocytes in almost all the hepatic lobules (Photos 4 and 5) and many pyknotic nuclei. These changes were more extensive than those seen in rats on the cholesterol diet alone.

\section{Correlation between changes in serum and liver cholesterol levels and fatty acid composition}

Figure 4 shows that the concentration of serum cholesterol is positively correlated with that of liver cholesterol in all the experimental diet groups $(r=0.720)$. The PUFA/SFA ratio for the serum also shows a positive correlation to the PUFA/SFA ratio for the liver $(r=0.551$, Fig. 5). These results clearly demonstrate that both changes in cholesterol level and PUFA/SFA ratio for the serum are consistent with the changes for the liver. Thus, the effects of a diet on changes in cholesterol level and fatty acid composition for the liver can be monitored by the changes in these parameters for the serum. Comparison of the results in Figs. 1, 3, 4 and 5 confirms that the liver lipid concentrations varied with the amounts of the phytosterol and PUFA added to a cholesterol diet, as well as those in serum. Dietary phytosterol and PUFA intake suppressed elevation of 


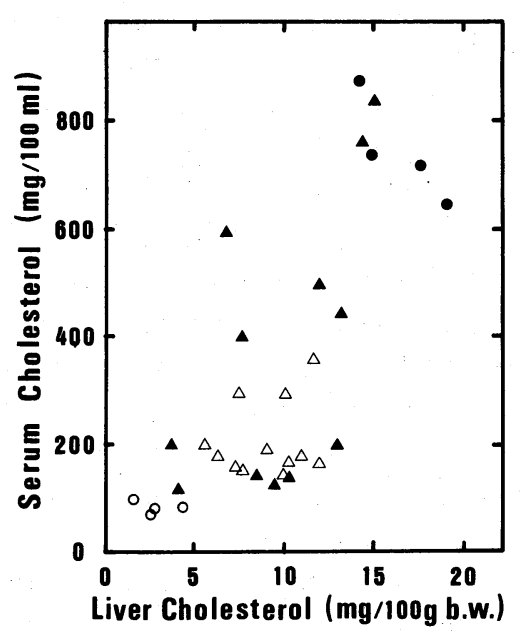

Fig. 4. Relationship between serum cholesterol and liver cholesterol levels in rats fed on different diets. For symbols, refer to Fig. $1(r=0.720, p<0.001)$.

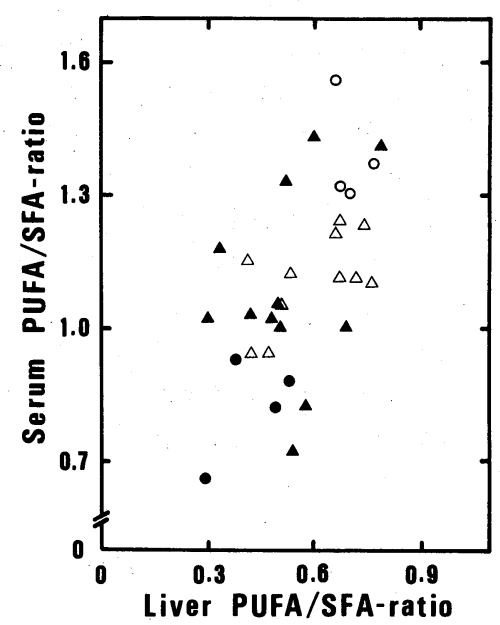

Fig. 5. Relationship between serum and liver PUFA/SFA ratios for rats fed on different diets. For symbols, refer to Fig. $1(r=0.521, p<0.01)$.

Photo 1. In the cholesterol diet group (group I in Table 1), moderate lipid droplets were found in hepatocytes of the peripheral zone. H-E stain, $\times 274$.

Photos 2 and 3. Light micrographs of liver cells of a rat on phytosterol-supplemented cholesterol diet (group $\mathbf{J}$ in Table 1). Relatively intact hepatocytes without lipid deposition. H-E stain; $2, \times 274 ; 3, \times 548$.

Photos 4 and 5. In the PUFA-fat diet group (group K in Table 1), foamy vacuolated hepatocytes with pyknotic nuclei were seen in the periportal area of the rat liver. HE stain; $4, \times 274 ; 5, \times 548$. 

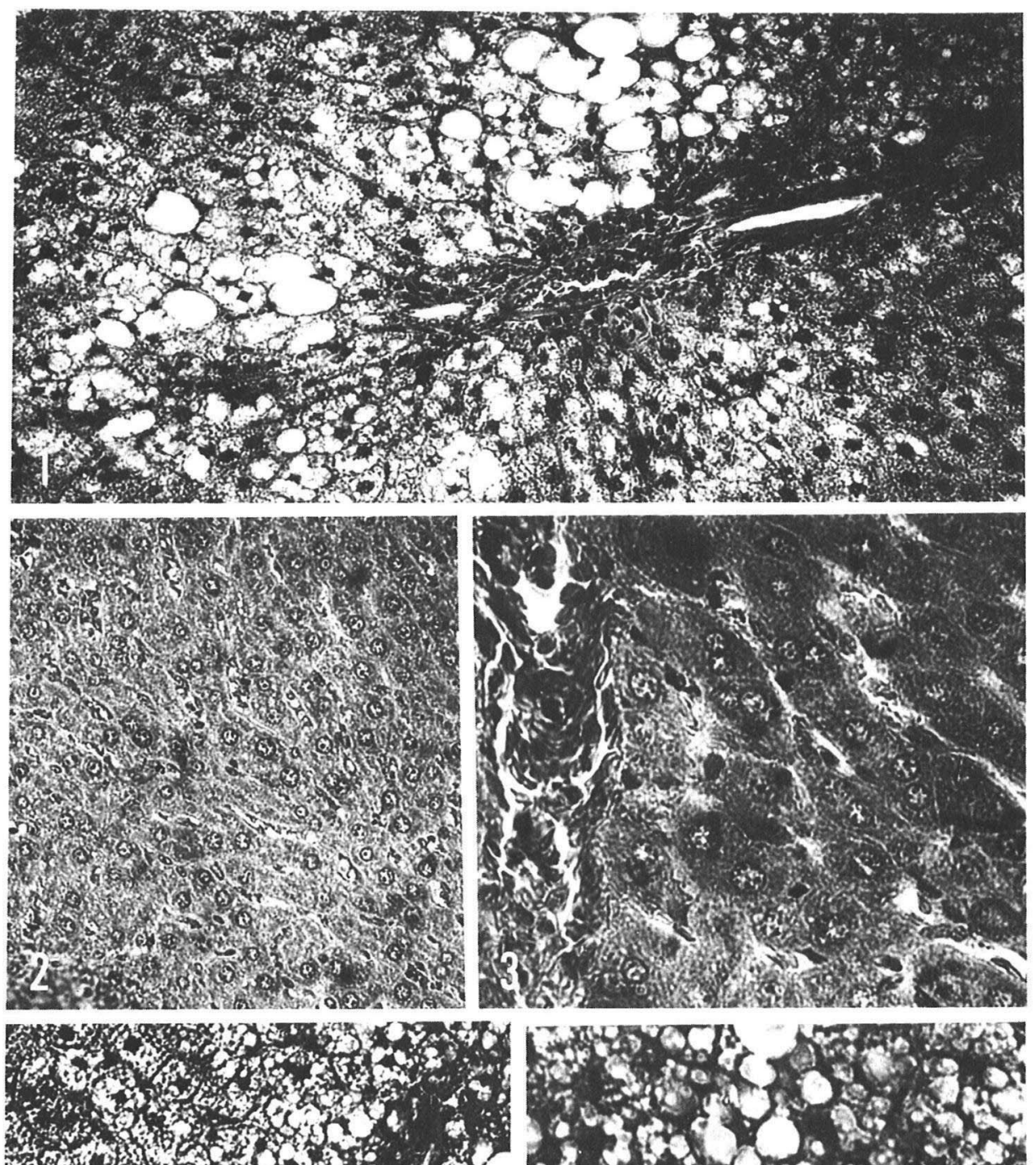

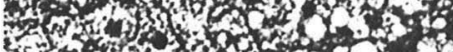

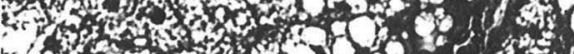

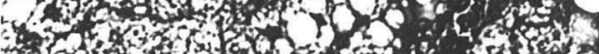
Hon

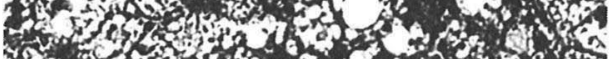

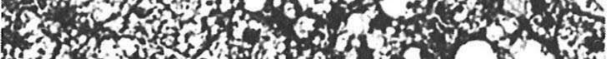

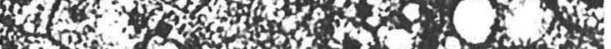

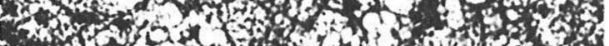

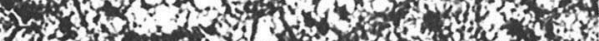

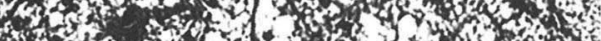

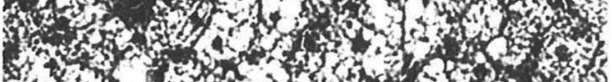
a)

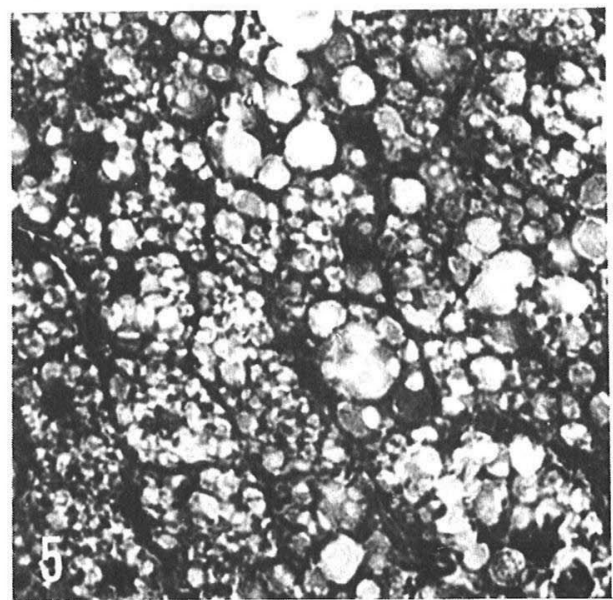


cholesterol levels in the serum and liver in this experimental study. This means that the influence of dietary cholesterol on the cholesterol level in the serum and liver varied with changes in the other components of the diet. The cholesterol levels in serum and liver seem to reflect cholesterol metabolism in the liver rather than the amount of dietary cholesterol. This may indicate that a hypocholesterolemic effect of both phytosterol and PUFA plays an importnt role in cholesterol metabolism which can be regulated not only by the hepatic synthesis of cholesterol but also by the biliary excretion of sterols and bile acids (10). Although the mechanism is uncertain, the metabolic interactions between the fatty acid composition and cholesterol level in both liver and serum have been clearly demonstrated in this work.

The authors gratefully acknowledge Riken Vitamin Co. for providing phytosterol and cod liver oil. This work was supported in part by grant No. 57580039 awarded by the Japan National Grant in Aid for Scientific Research in 1983.

\section{REFERENCES}

1) Kannel, W. B., Castelli, W. P., Gordon, T., and McNamara, P. M. (1971): Serum cholesterol, lipoproteins, and the risk of coronary heart disease, The Framingham study. Ann. Intern. Med., 74, 1-12.

2) Carlson, L. A., and Böttiger, L. E. (1972): Ischaemic heart-disease in relation to fasting values of plasma triglycerides and cholesterol, Stockholm prospective study. Lancet, 1, 865-868.

3) Kinsell, L. W., Partridge, J., Boling, L., Margen, S., and Michaels, G. (1952): Dietary modification of serum cholesterol and phospholipid levels. J. Clin. Endocrinol., 12, 909-913.

4) Connor, W. E., Stone, D. B., and Hodges, R. E. (1964): The interrelated effects on dietary cholesterol and fat upon human lipid levels. J. Clin. Invest., 43, 1691-1696.

5) Beveridge, J. M. R., Connell, W. F., Mayer, G. A., Firstbrook, J. B., and DoWolfe, M. S. (1955): The effects of certain vegetable and animal fats on the plasma lipids of humans. J. Nutr., 56, 311-319.

6) Ahrens, E. H., Jr., Insull, W., Jr., Blomstrand, R., Hirsch, J., Tsaltas, T. T., and Peterson, M. L. (1957): The influence of dietary fat on serum lipid levels in man. Lancet, 1, 943-953.

7) Spritz, N., and Mishkel, M. A. (1969): Effects of dietary fats on plasma lipid and lipoproteins: An hypothesis for the lipid-lowering effect of unsaturated fatty acids. $J$. Clin. Invest., 48, 78-86.

8) Anderson, J. T., Grande, R., and Keys, A. (1976): Independence of the effects of cholesterol and degree of saturation of the fat in the diet on serum cholesterol in man. Am. J. Clin. Nutr., 29, 1184-1189.

9) Toussant, M. J. Wilson, M. D., and Clarke, S. D. (1981): Coordinate suppression of liver acetyl-CoA carboxylase and fatty acid synthetase by polyunsaturated fat. $J$. Nutr., 111, 146-153.

10) Ramesha, C. S., Paul, R., and Ganguly, J. (1980): Effect of dietary unsaturated oils on the biosynthesis of cholesterol, and on biliary and fecal excretion of cholesterol and bile acids in rat. J. Nutr., 110, 2149-2158. 
11) O’Brien, B. C., Skutches, C. L., Henderson, G. R., and Reiser, R. (1977): Interrelated effects of food lipids on steroid metabolism in rats. J. Nutr., 107, 1444-1454.

12) Davis, W. W. (1955): Symposium on sitosterol III. The physical chemistry of cholesterol and $\beta$-sitosterol related to the intestinal absorption of cholesterol. Trans. $N$. Y. Acad. Sci., 18, 123-128.

13) Mattson, F. H., Volpenhein, R. A., and Erickson, B. A. (1977): Effect of plant sterol esters on the absorption of dietary cholesterol. J. Nutr., 107, 1139-1146.

14) Gerson, T., and Shorland, F. B. (1965): The effect of $\beta$-sitosterol on the metabolism of cholesterol and lipid in rats on a diet containing coconut oil. Biochem. J., 96, 399-403.

15) Konlande, J. E., and Fisher, H. (1969): Evidence for a nonabsorption antihypercholesterolemic action of phytosterol in the chiken. J. Nutr., 98, 435-442.

16) von Lossonczy, T. O., Ruiter, A., Bronsgeest-Schoute, H. C., van Gent. C. M., and Hermus, R. J. J. (1978): The effect of a fish diet on serum lipids in healthy human subjects. Am. J. Clin. Nutr., 13, 1340-1346.

17) Peifer, J. J., Janssen, F., Ahn, P., Cox, W., and Lundberg, W. O. (1960): Studies on the distribution of lipoids in hypercholesterolemic rats. 1. The effect of feeding palmitate, linoleate, menhades, and tuna oil. Arch. Biochem. Biophys., 86, 302-308.

18) Hirai, K., Ohno, Y., Nakano, T., and Izutani, K. (1984): Effects of dietary fats and phytosterol on serum fatty acid composition and lipoprotein cholesterol in rats. $J$. Nutr. Sci. Vitaminol. 30, 101-112.

19) Harper, A. E. (1959): Amino acid balance and imbalance, part I. Dietary level of protein and amino acid imbalance. J. Nutr., 68, 405-424.

20) The Japan Oil Chemist's Society (1977): Standard Method for Analysis of Fats and Oils (in Japanese). No. 2.4.10-71.

21) Metcalf, L. D., and Schmitz, A. A. (1961): The rapid preparation of fatty acid esters for gas chromatographic analysis. Anal. Chem., 33, 363-364.

22) Folch, J., Lees, M., and Soloane-Stanley, G. H. (1957): A simple method for the isolation and purification of total lipids from animal tissues. J. Biol. Chem., 226, 497-509.

23) Shibahara, A., Yoshida, H., and Kajimoto, G. (1978): Gas chromatographic analysis of fatty acid methyl esters on SP-2300 liquid phase. Yukagaku (in Japanese), 27, 233-236.

24) Miwa, T. K., Milolajczak, K. L., Earle, F. R., and Wolffe, J. A. (1960): Gas chromatographic characterization of fatty acids-identification of constants for mono- and dicarboxylic methyl esters. Anal. Chem., 32, 1739-1742.

25) Sano, Y. (1970): Histochemistry, Principle and Technical Methods, 3rd ed., Nanzan-do Corp., Tokyo, pp. 79-141, 179-197, 476-477.

26) Beveridge, J. M. R., Connell, W. F., Mayer, G. A., and Haust, H. L. (1960): The response of man to dietary cholesterol. J. Nutr., 71, 61-65.

27) Jackson, R. L., Taunton, O. D., Morrisett, U. D., and Gotto, A. M. (1978): The role of dietary polyunsaturated fat in lowering blood cholesterol in man. Circ. Res., 42, 447-453.

28) Okey, R., Lyman, M. M., Harris, A. G., Einset, B., and Hain, W. (1959): Dietary fat and cholesterol metabolism: Effects of unsaturation of dietary fats on liver and serum lipids. Metabol. Clin. Exp., 8, 241-255. 